\title{
A Cross-Sectional Web Survey of Satisfaction with Treatment for Pain in Participants with Suspected Diabetic Peripheral Neuropathic Pain in Both Feet
}

\author{
Takahisa Deguchi (D) - Hiroshi Takatsuna · Mizuka Yokoyama • \\ Kazuhito Shiosakai · Teruyoshi Inoue (D) - Haruhiko Seki · \\ Yoshiyuki Uetake
}

Received: March 29, 2021 / Accepted: May 27, 2021 / Published online: July 8, 2021

(c) The Author(s) 2021

\section{ABSTRACT}

Introduction: Diabetic peripheral neuropathic pain (DPNP), a symptom of diabetic polyneuropathy (DPN), is underdiagnosed in people with diabetes. To date, no studies have determined the relationship between diagnosis of DPN and satisfaction with treatment for pain. Additionally, the factors that influence satisfaction with treatment for pain remain unknown. This questionnaire study was conducted to understand satisfaction with treatment for pain among participants with diabetes who experienced bilateral pain or numbness in their feet.

Supplementary Information The online version contains supplementary material available at https:// doi.org/10.1007/s12325-021-01810-x.

T. Deguchi $(\bowtie)$

Department of Diabetes and Endocrine Medicine, Kagoshima University Graduate School of Medical and Dental Science, Kagoshima, Japan

e-mail: degdeg@m3.kufm.kagoshima-u.ac.jp

H. Takatsuna · M. Yokoyama · T. Inoue Medical Affairs Division, Daiichi Sankyo Co., Ltd., Tokyo, Japan

K. Shiosakai

Digital Transformation Management Division,

Daiichi Sankyo Co., Ltd., Tokyo, Japan

H. Seki · Y. Uetake

INTAGE Healthcare Inc., Tokyo, Japan
Methods: This cross-sectional, observational, web-based questionnaire study for participants with diabetes and suspected DPNP was conducted in Japan. Potential respondents were registered in the INTAGE Disease Panel or the Rakuten Insight Disease Panel. The primary endpoint was the number and percentage of participants who were satisfied with their DPNP treatment. Secondary endpoints included participant opinions regarding treatment-related efficacy, side effects, and economic burden, and factors affecting satisfaction with treatment.

Results: The questionnaire was accessed by 7565 potential participants; 777 met the eligibility criteria (final analysis set). Satisfaction with treatment for bilateral foot pain was low (satisfied, 27.9\%; neither satisfied nor unsatisfied, $42.2 \%$; unsatisfied, $23.4 \%$; very unsatisfied, 6.4\%). Participants were somewhat more satisfied with treatment side effects than with treatment efficacy and economic burden. Satisfaction with treatment mainly differed by improvement in actions in daily life, improvement in quality of life, and communication with doctors. The diagnostic testing rate for DPN was low, and diagnosis was more common in participants who complained of symptoms of pain and numbness (any visit) versus those who did not.

Conclusion: Participants with diabetes who experience bilateral foot pain or numbness reported a low level of satisfaction with treatment for pain. 


\section{PLAIN LANGUAGE SUMMARY}

People with diabetes may develop diabetic polyneuropathy and experience diabetic peripheral neuropathic pain, which is often felt as pain or numbness below the knee. This study aimed to learn whether participants with diabetes who had pain or numbness in both feet were satisfied with the pain treatment they received. Factors affecting satisfaction with treatment were also evaluated. Potential participants with diabetes identified from two commercial databases (INTAGE Disease Panel or Rakuten Insight Disease Panel) of patients with various diseases living in Japan were asked to respond to our web survey. Besides satisfaction with treatment for pain, participants were asked about how well their treatment was working, treatment side effects, how treatment affected them financially, and what factors affected their satisfaction with treatment. The main finding was that only $27.9 \%$ of participants were satisfied with their treatment for foot pain and numbness. Generally, participants were more satisfied with treatment side effects than they were with how well the treatment worked, and how treatment affected them financially. Participants were more satisfied if they had an improved ability to perform everyday activities or experienced an improvement in quality of life with treatment. Participants were also more satisfied if they communicated well with their physician. The rate of diagnostic tests was low; however, participants were more likely to receive a diagnostic test when they complained of pain or numbness than when they did not. On the basis of these findings, we think improvements in the treatment of foot pain or numbness in those with diabetes are needed.

Keywords: Diabetic peripheral neuropathic pain; Diagnosis; DN4; Satisfaction with treatment for pain; Web survey

\section{Key Summary Points}

Why carry out this study?

It is estimated that approximately $6-37 \%$ of people with diabetes have diabetic peripheral neuropathic pain (DPNP)

However, the diagnosis rate for DPNP is low, and treatment for this condition has not progressed, suggesting a low level of satisfaction with treatment in people with DPNP

Using a web-based questionnaire, this study aimed to examine satisfaction with treatment for bilateral foot pain in participants with diabetes who experienced bilateral pain or numbness in their feet

What was learned from the study?

Satisfaction with treatment for pain for participants with diabetes who had pain or numbness in both feet was $27.9 \%$

Appropriate diagnosis and treatment of DPNP and good communication with doctors are needed to improve satisfaction with treatment in these participants

\section{DIGITAL FEATURES}

This article is published with digital features, including a summary slide and plain language summary, to facilitate understanding of the article. To view digital features for this article go to https://doi.org/10.6084/m9.figshare. 14681004 .

\section{INTRODUCTION}

One common complication of diabetes mellitus is diabetic polyneuropathy (DPN), a major type of diabetic peripheral neuropathy [1]. Diabetic peripheral neuropathic pain (DPNP) is a 
symptom of DPN that often presents as abnormal sensations, numbness, and pain generally below the knee and in both legs [2]. Along with these symptoms, patients may experience problems with sleep, anxiety, depression, and a reduced quality of life (QOL) [3-5]. DPNP is reported to occur in those with diabetes mellitus at an incidence rate of $28 \%$ in the USA [6, 7], between $6 \%$ and $34 \%$ in Europe [8], and between $28 \%$ and $37 \%$ in Japan [9-11].

While there is no universal diagnostic standard for DPN, the USA [12], Europe [13], and Japan all use an autonomic nerve function test and a nerve conduction test to make a definitive diagnosis. The Japanese Study Group of Diabetic Neuropathy developed a set of simple diagnostic criteria in which patients are tested for (1) subjective symptoms that may occur as a result of DPN, (2) decreased or absent bilateral Achilles tendon reflex, and (3) decreased bilateral internal ankle vibration sensation [14]. If patients test positive for two or more of these, they are considered to have DPN. Similarly, the Japan Diabetes Society recommends simple diagnostic examinations for DPN, such as vibration sensation and Achilles tendon reflex tests [15]. Because such tests are time consuming and require mastery of the procedure, it is likely that some cases may be difficult to diagnose in diabetes outpatient clinics.

Some studies have indicated low implementation rates of diagnostic tests in Japan and low physician awareness of DPN in patients, suggesting that DPNP is underdiagnosed $[16,17]$. A large-scale survey of approximately 200,000 participants with diabetes was conducted in Japan between 2006 and 2007 to learn more about symptoms that patients experienced in their feet [16]. This study found that $18.9 \%$ of survey participants experienced a tingling sensation in their toes and $20.1 \%$ experienced numbness in their toes. Regarding DPN diagnostic tests, only $68.2 \%$ and $36.1 \%$ of participants had been given Achilles tendon reflex or vibration sensation tests, respectively, indicating that not all participants with diabetes were given the tests needed to diagnose DPN.

Sasaki et al. recently performed a web survey to investigate the real-world status of bilateral foot pain in participants being treated for diabetes in Japan [18]. Of the 7754 survey respondents, $22.8 \%$ had bilateral foot pain symptoms. Among those with symptoms, $44.7 \%$ did not consult a doctor for their pain, $25.9 \%$ had problems in their daily life due to their foot pain, and $81.8 \%$ did not know that there were treatments available to improve symptoms of foot pain.

While there is a clear burden of bilateral foot pain in patients being treated for diabetes, neither the relationship between the diagnosis of DPNP and satisfaction with treatment for pain nor the factors that influence satisfaction with treatment for pain have been elucidated. A retrospective study conducted in the USA found that up to half of participants who were being treated for DPNP discontinued their initial treatments within 3 months of initiation, suggesting a low level of satisfaction with treatment and/or poor tolerability [19]. This result and those from our previous web-based survey study indicate that patient satisfaction with treatment for bilateral foot pain symptoms may be insufficient.

It is important to understand that DPNP is an underdiagnosed condition and that patients are likely unsatisfied with treatment for foot pain. This understanding can help inform medical professionals who treat those with diabetes, which can lead to improvements in the diagnosis and treatment of DPNP and, subsequently, in QOL. This observational, web-based study was conducted to examine satisfaction with treatment for bilateral foot pain in participants with diabetes who experienced bilateral pain or numbness in their feet. Factors affecting satisfaction with treatment for pain such as treatment efficacy, side effects, and economic burden; communication with physicians; satisfaction with daily life and QOL; treatment preferences; diagnostic testing; and timing of diagnostic testing were also evaluated.

\section{METHODS}

\section{Study Design}

This was a cross-sectional, observational, webbased questionnaire study for participants with suspected DPNP. The study questionnaire was designed to collect information on what 
participants with suspected DPNP thought about various aspects of their care, including satisfaction with treatment efficacy, side effects, and economic burden. The specific questions asked in the present study were based on those asked in previously published survey studies of satisfaction with treatment for patients with psoriasis or chronic pain [20, 21]. Questions from the Douleur Neuropathique 4 (DN4) questionnaire, which is a screening tool for neuropathic pain, were also included [22, 23].

The study protocol was approved by the ethics review board at the Kitamachi Clinic prior to conducting the study (August 19, 2020). All procedures were conducted in accordance with the ethical standards of the responsible committee on human experimentation, both institutional and national, and/or with the Helsinki Declaration of 1964 and later versions. This study was also conducted in accordance with the Japanese ethical guidelines for medical and health research involving human subjects [24], the Act on the Protection of Personal Information, and the revised Personal Information Protection Act. The study was registered with the University hospital Medical Information Network Clinical Trials Registry (UMIN000041981). All participants provided electronic informed consent to participate.

\section{Participants}

Participants with diabetes who were registered in the INTAGE Disease Panel (INTAGE Inc., Tokyo, Japan) [25] or those with type 1 diabetes, type 2 diabetes, diabetic nephropathy, or diabetic neuropathy who were registered in the Rakuten Insight Disease Panel (Rakuten Insight, Inc., Tokyo, Japan) [26] were eligible to participate in the study. The composition ratio of the gender and age of participants with diabetes (type 1 , type 2 , other) was calculated using the values reported in a patient survey conducted by the Ministry of Health, Labor and Welfare of Japan [27] as reference values. The target number of participants for each gender and age was set so that the distribution of gender and age would match as closely as possible to the reference values. This information was used to randomly extract eligible participants from the disease panel, and survey invitations were distributed. Randomization was carried out using software specifically utilized by each of the companies providing the panels (INTAGE Inc. and Rakuten Insight, Inc.); however, the details cannot be disclosed. This process was utilized for all eligible potential participants with diabetes mellitus who received an invitation to participate.

After eligible participants received the survey invitation, they were screened using the following inclusion and exclusion criteria. Participants were included if they were at least 20 years old, were receiving outpatient treatment for diabetes at the time of study registration, had pain or numbness in both feet and had consulted a doctor about those symptoms, and were able to comprehend and appropriately answer the study questions. While a confirmation of self-reported diabetes diagnosis was not required, outpatient treatment for diabetes implied a definitive diagnosis by a medical professional. Participants were excluded if they were involved in market research or advertising/marketing, were members of the news media, were healthcare providers, or were employees of pharmaceutical companies. The exclusion criteria were intended to minimize the possibility of false responses, to limit bias, and to improve the reliability of the survey results. Potential respondents who met all the inclusion criteria and none of the exclusion criteria and who provided electronic informed consent were included in the study.

\section{Data Collection and Management}

Study data were collected between November 9 and 16, 2020 using a one-time web questionnaire that was expected to take approximately 10 min to complete. Anonymity of respondents was required by INTAGE Inc. and Rakuten Insight, Inc. An email containing the web link to the web-based questionnaire was sent to each potential participant. The questionnaire was written and conducted in Japanese. Participants filled in the questionnaire as a self-completion survey with instructions provided for each 
question, and response data were recorded and collected electronically and stored in a database.

Data collection was concluded after the target number of responses was reached and confirmed by INTAGE Healthcare Inc. and approved by Daiichi Sankyo Co., Ltd (Tokyo, Japan). Collected data were compiled, cleaned, and reviewed by INTAGE Healthcare Inc., followed by final confirmation and database lock by the data manager and statistical analysis manager. No data corrections were allowed after the database lock. This survey was designed so that participants were unable to submit their answers unless they completed the questionnaire in a fixed order. Therefore, there were no missing data.

\section{Study Endpoints}

The primary endpoint was the number and percentage of participants who reported satisfaction with treatment for bilateral foot pain based on a scale of 1 (very satisfied) to 5 (very unsatisfied). The secondary endpoints included the number and percentage of participants who reported (1) satisfaction with treatment for bilateral foot pain in regard to efficacy (Q13-1), side effects (Q13-2), and economic burden (Q13-3) (from 1 [very satisfied] to 5 [very unsatisfied]); (2) degree of improvement in actions in daily life (ADL) provided by treatment for bilateral foot pain (Q15, from 1 [very well] to 5 [very poor]); (3) improvement in QOL provided by treatment for bilateral pain (Q16, from 1 [very well] to 5 [very poor]); (4) degree of communication with doctors (Q17, from 1 [very well] to 5 [very poor]); (5) participant understanding regarding the cause of DPNP symptoms $(\mathrm{Q} 20$, symptoms due to DPN, orthopedic disease, or other); and (6) participant preference for bilateral foot pain treatment $(\mathrm{Q} 22$, relative to current treatment: stronger treatment, continuation of current treatment, or weaker treatment). For the secondary endpoints, the first four questions listed had five levels of responses and the final two questions listed had three possible options. Other endpoints included subgroup analysis of satisfaction with treatment for bilateral foot pain according to selected baseline and disease characteristics.

Subgroup analysis for satisfaction with treatment for bilateral foot pain was conducted using the following stratification factors: gender, age, duration of bilateral foot pain, improvement of ADL by treatment for bilateral foot pain (Q15), improvement in QOL by treatment for bilateral foot pain (Q16), degree of communication with doctors (Q17), presence of a treatment plan, preference for bilateral foot pain treatment $(\mathrm{Q} 22)$, groups with $\geq 3$ or $<3$ "yes" in the DN4 questionnaire (in total and according to whether the participant received treatment; a score of $\geq 3$ in the DN4 questionnaire is the criterion for determining whether DPNP is suspected), "yes" or "no" group for each item in the DN4 questionnaire, method of DPN diagnosis, timing of DPN diagnosis, Numerical Rating Scale (NRS) score groups (1-3, 4-6, and 7-10), duration of diabetes, recent glycated hemoglobin $(\mathrm{HbA} 1 \mathrm{c})$ value, and type of treatment for pain.

\section{Statistical Methods}

To achieve the maximum width of the 95\% confidence interval (CI) of each survey question within $\pm 5 \%$, it was determined that 400 participants were needed for the analysis set. The analysis set consisted of participants who met all of the inclusion criteria and none of the exclusion criteria, and provided electronic informed consent.

Summary statistics were calculated for continuous variables, and calculations of the number and percentage of participants were used to summarize categorical variables related to participant background. For the primary endpoint, the percentage of participants who reported satisfaction with treatment was calculated using the following formula: (Very satisfied + Satisfied)/(Number of cases to be analyzed) $\times 100(\%)$. The $95 \%$ CIs were also calculated. No hypothesis test was performed as this was a survey of the current status of participants suspected to have DPNP.

Subgroup analysis for satisfaction with treatment for bilateral foot pain was conducted 
in the same manner as that of the whole population. All statistical analyses were performed using SAS version 9.4 (SAS Institute, Inc., Cary, NC, USA).

\section{RESULTS}

\section{Participant Characteristics}

Of the 7565 potential participants with diabetes mellitus who accessed the questionnaire, 6430 provided electronic informed consent and 777 met the inclusion criteria and did not meet any of the exclusion criteria and responded to the questionnaire (analysis set). Almost half of the participants included in the present study were also included in a previously published web survey that utilized the same patient panels [18]. Participant characteristics are shown in Table 1 . The percentage of male participants was $69.8 \%$, the most common age group was 60-69 years old (29.9\%), and most participants had type 2 diabetes (90.6\%). Overall, $18.7 \%$ of participants were diagnosed with diabetes within the last 5 years and $38.7 \%$ were diagnosed at least 15 years ago. The most recent HbA1c value was $6.5 \%$ to $<7.0 \%$ for $24.7 \%$ of participants, which is within the target range for HbA1c set for the prevention of complications by the Japan Diabetes Society [28]. Most participants were treated for their pain at a clinic $(63.6 \%)$ or a department of diabetes (51.2\%).

Pain-related characteristics of the participants are shown in Table 2. Most participants (71.7\%) had experienced bilateral foot pain for at least 1 year, with the most common symptoms of pain (DN4 questionnaire) being numbness $(75.5 \%)$, tingling $(48.9 \%)$, and electric shocks $(41.1 \%)$. Oral medication was the most common type of treatment for pain $(49.5 \%)$. The mean \pm standard deviation (SD) NRS, which reflects current pain and numbness, was $4.07 \pm 2.35$. There were 337 (43.4\%) participants who answered "yes" to at least three questions on the DN4 questionnaire. The most common method to diagnose DPN was reported as "complaints of pain or numbness" (73.5\%), with other diagnostic tests used for less than
Table 1 Participant background characteristics

\begin{tabular}{lc}
\hline Characteristic & $\begin{array}{c}\text { Participant } \\
(\boldsymbol{n}=777)\end{array}$ \\
\hline Gender & \\
Male & $542(69.8)$ \\
Female & $235(30.2)$ \\
Age, years & \\
$20-29$ & $3(0.4)$ \\
$30-39$ & $31(4.0)$ \\
$40-49$ & $92(11.8)$ \\
$50-59$ & $222(28.6)$ \\
$60-69$ & $232(29.9)$ \\
$70-79$ & $170(21.9)$ \\
$\geq 80$ & $27(3.5)$
\end{tabular}

Type of diabetes

Type 1

Type 2

$704(90.6)$

Unknown

$26(3.3)$

Most recent $\mathrm{HbAlc}$ value, \%

$<6.0$

6.0 to $<6.5$

$124(16.0)$

6.5 to $<7.0$

$192(24.7)$

7.0 to $<7.5$

$173(22.3)$

7.5 to $<10.0$

$160(20.6)$

$\geq 10.0$

$28(3.6)$

Unknown

$36(4.6)$

Duration of diabetes, years

$<5$

$145(18.7)$

5 to $<10$

$179(23.0)$

10 to $<15$

143 (18.4)

$\geq 15$

$301(38.7)$

Unknown

$9(1.2)$

Comorbidities

Dyslipidemia

$256(32.9)$

Hypertension

$418(53.8)$ 
Table 1 continued

\begin{tabular}{|c|c|}
\hline Characteristic & $\begin{array}{l}\text { Participants } \\
(n=777)\end{array}$ \\
\hline \multicolumn{2}{|l|}{ Outpatient status } \\
\hline Going to hospital & $771(99.2)$ \\
\hline On a home-visit basis & $10(1.3)$ \\
\hline Hospitalized & $4(0.5)$ \\
\hline \multicolumn{2}{|l|}{ Clinical department } \\
\hline Diabetes & $398(51.2)$ \\
\hline Internal medicine & $336(43.2)$ \\
\hline Other & $43(5.5)$ \\
\hline \multicolumn{2}{|l|}{ Medical institution } \\
\hline Clinic & $494(63.6)$ \\
\hline University hospital & $73(9.4)$ \\
\hline $\begin{array}{l}\text { Hospital other than university } \\
\text { hospital }\end{array}$ & $207(26.6)$ \\
\hline Home-visit nursing station & $0(0.0)$ \\
\hline Healthcare facility for the elderly & $1(0.1)$ \\
\hline Other & $2(0.3)$ \\
\hline \multicolumn{2}{|l|}{ Frequency of alcohol consumption } \\
\hline Do not drink & $390(50.2)$ \\
\hline Drink occasionally & $247(31.8)$ \\
\hline Drink every day & $140(18.0)$ \\
\hline \multicolumn{2}{|l|}{ Smoking status } \\
\hline Never & $416(53.5)$ \\
\hline Former smoker & $173(22.3)$ \\
\hline Current smoker & $188(24.2)$ \\
\hline
\end{tabular}

Data are presented as $n$ (\%) unless otherwise noted HbAlc glycated hemoglobin

$50 \%$ of participants and nerve conduction tests used in only $18.1 \%$ of participants. Neuropathy diagnostic testing of all types was performed more often when the participant complained of
Table 2 Pain-related participant characteristics

\begin{tabular}{lc}
\hline Characteristic & $\begin{array}{c}\text { Participants } \\
(\boldsymbol{n}=777)\end{array}$ \\
\hline Duration of bilateral foot pain, years \\
$<0.5$ & $72(9.3)$ \\
0.5 to $<1.0$ & $83(10.7)$ \\
$\geq 1.0$ & $557(71.7)$ \\
Unknown & $65(8.4)$
\end{tabular}

Symptoms of pain according to the DN4 questionnaire

Burning sensation, yes/no

$99(12.7) / 678(87.3)$

Painful cold, yes/no

$245(31.5) / 532(68.5)$

Electric shocks, yes/no

$319(41.1) / 458(58.9)$

Tingling, yes/no

$380(48.9) / 397$ (51.1)

Pins and needles, yes/no

170 (21.9)/607 (78.1)

Numbness, yes/no

$587(75.5) / 190$ (24.5)

Itching, yes/no

$161(20.7) / 616$ (79.3)

DN4 questionnaire

"Yes" to $\geq 3$

$337(43.4)$

"Yes" to $<3$

$440(56.6)$

Diagnosis of neuropathy

Achilles tendon reflex test

Received

$359(46.2)$

Never received

$374(48.1)$

Unknown

$44(5.7)$

Vibration sensation test

Received

244 (31.4)

Never received

475 (61.1)

Unknown

$58(7.5)$

Pain sensation test

Received $270(34.7)$

Never received 438 (56.4)

Unknown

$69(8.9)$ 
Table 2 continued

\begin{tabular}{ll}
\hline Characteristic & $\begin{array}{l}\text { Participants } \\
(n=777)\end{array}$
\end{tabular}

Complaints of pain or numbness

Received

$571(73.5)$

Never received

$157(20.2)$

Unknown

$49(6.3)$

Autonomic nerve function test

Received

$305(39.3)$

Never received

409 (52.6)

Unknown

$63(8.1)$

Nerve conduction test

Received

$141(18.1)$

Never received

$575(74.0)$

Unknown

$61(7.9)$

Timing of diagnosis

Achilles tendon reflex test

At first visit

$126(16.2)$

When complaining of pain or numbness

Regular

$152(19.6)$

Unknown

$53(6.8)$

$61(7.9)$

Vibration sensation test

At first visit

$70(9.0)$

When complaining of pain or numbness

Regular

$109(14.0)$

Unknown

$46(5.9)$

$41(5.3)$

Pain sensation test

At first visit

$78(10.0)$

When complaining of pain or numbness

Regular

$132(17.0)$

Unknown

$50(6.4)$

$37(4.8)$

Complaints of pain or numbness

At first visit

$139(17.9)$
Table 2 continued

\begin{tabular}{ll}
\hline Characteristic & $\begin{array}{l}\text { Participan } \\
(n=777)\end{array}$ \\
\hline When complaining of pain or numbness & $335(43.1)$ \\
Regular & $135(17.4)$ \\
Unknown & $29(3.7)$ \\
Autonomic nerve function test & \\
At first visit & $77(9.9)$ \\
When complaining of pain or numbness & $107(13.8)$ \\
Regular & $99(12.7)$ \\
Unknown & $47(6.0)$
\end{tabular}

Nerve conduction test

At first visit

$44(5.7)$

When complaining of pain or numbness

$65(8.4)$

Regular

38 (4.9)

Unknown

$14(1.8)$

Type of treatment for pain

Oral medication

$385(49.5)$

Topical medication

$161(20.7)$

Injection

77 (9.9)

Non-pharmacotherapy

$57(7.3)$

None

303 (39.0)

NRS, mean \pm SD

$4.07 \pm 2.35$

Established treatment goal

$476(61.3)$

Adherence to antidiabetic drugs

Take as instructed by doctor

$665(85.6)$

Sometimes forget

98 (12.6)

Often forget

$6(0.8)$

Hardly take as instructed

$8(1.0)$

Duration of treatment for pain, years

$<0.5$

$61(7.9)$

0.5 to $<1.0$

$65(8.4)$ 
Table 2 continued

\begin{tabular}{ll}
\hline Characteristic & $\begin{array}{l}\text { Participants } \\
(\boldsymbol{n}=777)\end{array}$ \\
\hline$\geq 1.0$ & $325(41.8)$ \\
Unknown & $23(3.0)$ \\
\hline
\end{tabular}

Data are presented as $n$ (\%) unless otherwise noted

NRS Numerical Rating Scale, SD standard deviation, DN4

Douleur Neuropathique 4

pain or numbness at any visit. Most participants $(61.3 \%)$ had an established treatment goal.

\section{Study Endpoints}

\section{Primary Endpoint}

Regarding satisfaction with treatment for bilateral foot pain, $27.9 \%$ of participants $(95 \%$ CI $24.8 \%, 31.2 \%)$ were either "very satisfied" or "satisfied", 42.2\% were "neither (satisfied nor unsatisfied)", 23.4\% were "unsatisfied", and $6.4 \%$ were "very unsatisfied" (Fig. 1).

\section{Secondary Endpoints}

The results for the secondary endpoints are shown in Fig. 2. For all three questions regarding satisfaction with treatment (efficacy, Q13-1; side effects, Q13-2; economic burden, Q13-3), most participants answered "neither". For Q131 and Q13-3, the proportion of participants who answered either "satisfied" or "very satisfied" was lower than the proportion who answered either "unsatisfied" or "very unsatisfied". The opposite was true for Q13-2, with more participants being satisfied ("satisfied" and "very satisfied" combined) than unsatisfied ("unsatisfied" and "very unsatisfied" combined). Most participants reported that they did not experience any change ("neither") in ADL (Q15) or QOL (Q16) with the treatment provided. Of those who reported a change, more reported an improvement ("well" or "very well") than a reduction ("poor" or "very poor") in ADL and QOL. Most participants were satisfied (> 60\%; "well" (46.6\%) and "very well" (17.0\%) combined) with the level of communication they had with their doctor about their bilateral foot pain (Q17). Over half (56.4\%) of participants indicated that the cause of their bilateral foot pain was DPN (Q20). Regarding participants' opinion on their current treatment for bilateral foot pain (Q22), most (58.6\%) were happy to continue their current treatment; however, $32.0 \%$ wished to have a stronger treatment than they were currently receiving.

\section{Other Endpoints}

Subgroup analysis for satisfaction with treatment for bilateral foot pain according to select participant and disease characteristics is shown in the Supplementary Material and Tables 3 and 4. The results of satisfaction with treatment according to participant background and disease characteristics are shown in the Supplementary Material (Supplemental Table 1). Satisfaction with treatment was similar for males and females. Satisfaction among those in the older age groups (between 40 and at least 80 years old) was between $22.2 \%$ and $31.0 \%$. Satisfaction with treatment was somewhat lower among participants with a longer duration of pain (at least 1 year), and those with a low NRS score (1-3) were more satisfied with the treatment than those with higher scores. Participants who had a recent HbA1c value $\geq 6.5 \%$ were less satisfied with treatment provided than those with lower values. Among participants who had been diagnosed with diabetes for at least 5 years, satisfaction decreased with increased duration of diabetes. Participants who received injections for treatment of bilateral foot pain had a higher level of satisfaction with treatment than those who received other types of treatment; participants who were not receiving any treatment had the lowest level of satisfaction with treatment.

The results of satisfaction with treatment according to communication with doctors, ADL, QOL, and treatment preference are shown in Table 3. Satisfaction with treatment tended to be higher among participants who reported good communication with their doctor and among those who had an established treatment goal, which involves communication with doctors. Participants who reported improvements in ADL and QOL with treatment tended to have a higher level of satisfaction with treatment. Participants who reported that they 


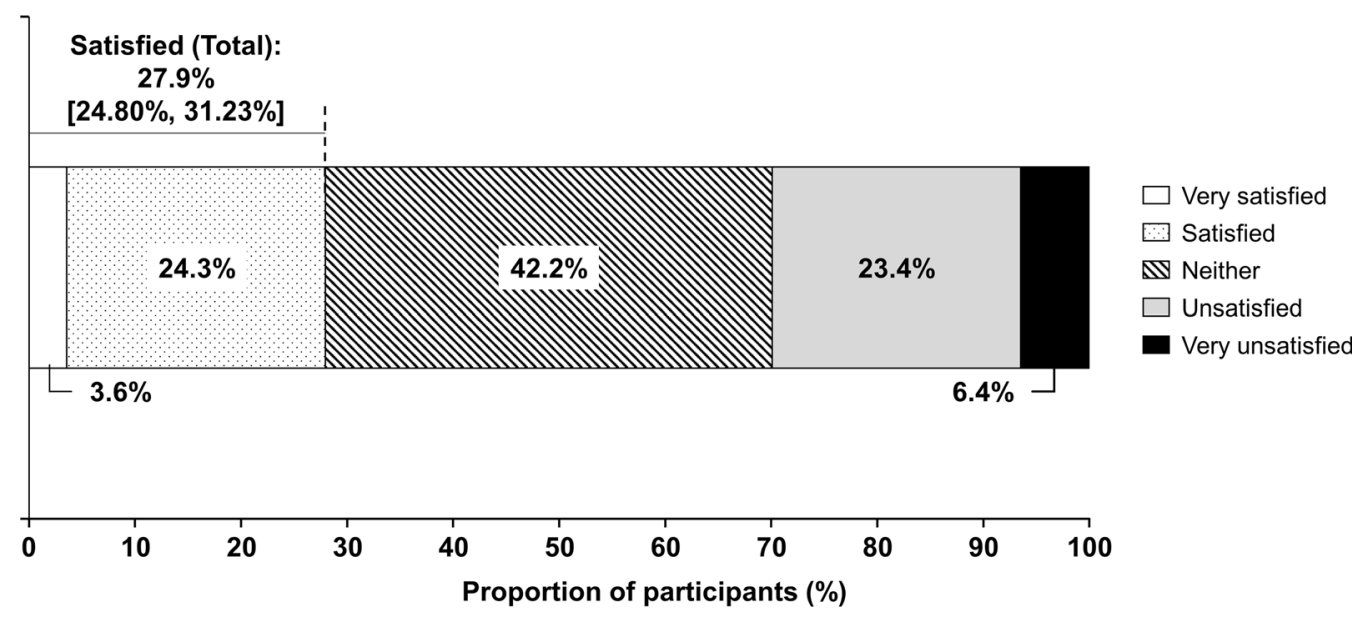

Fig. 1 Participant satisfaction for treatment of bilateral foot pain (primary endpoint). The value reported for "Satisfied (Total)" is the sum of "Very satisfied" and "Satisfied" responses. The 95\% confidence interval is reported for participants who were "Satisfied"

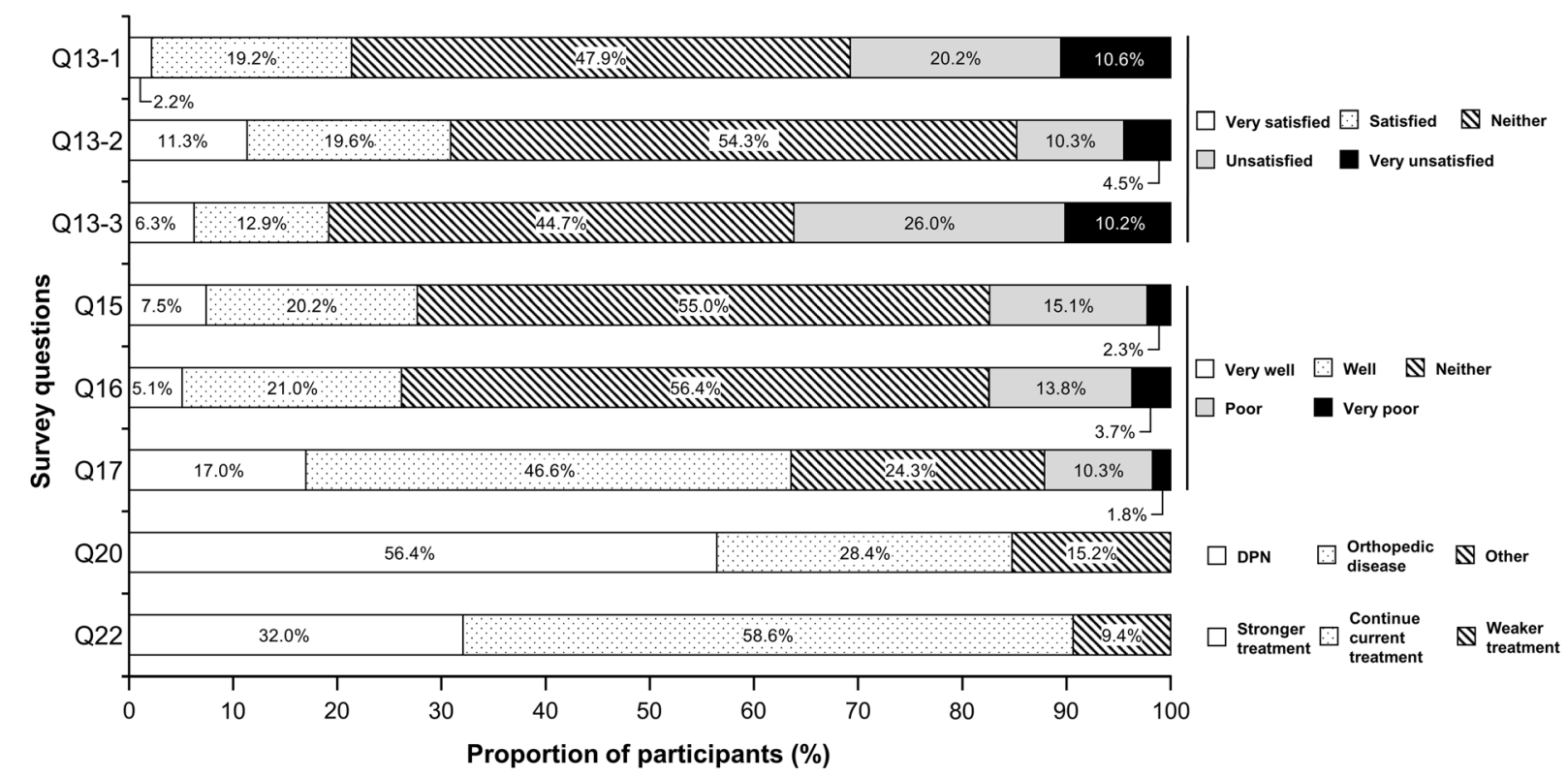

Fig. 2 Participant satisfaction for survey questions Q13, Q15-Q17, Q20, and Q22 (secondary endpoints). Q13-1: Satisfaction with treatment for bilateral foot pain regarding efficacy, Q13-2: Satisfaction with treatment for bilateral foot pain regarding side effects, Q13-3: Satisfaction with treatment for bilateral foot pain regarding economic burden, Q15: Improvement of ADL by treatment for bilateral foot pain, Q16: Improvement of QOL by treatment for bilateral foot pain, Q17: Degree of communication with doctors, Q20: Understanding of the cause of pain symptoms, Q22: Preference for bilateral foot pain treatment. ADL actions in daily life, DPN diabetic polyneuropathy, QOL quality of life

wished to have stronger treatment than they were currently receiving tended to be less satisfied than those who preferred to continue their current treatment or receive weaker treatment.
Satisfaction with treatment according to DN4 questionnaire answers is shown in Table 4. Participants who answered "yes" to at least three questions on the DN4 questionnaire were less 
Table 3 Satisfaction with treatment by communication with doctors, ADL and QOL improvement, and preference for pain treatment

\begin{tabular}{|c|c|c|}
\hline & \multicolumn{2}{|l|}{ Satisfied $^{\mathrm{a}}$} \\
\hline & $n(\%)$ & $95 \% \mathrm{CI}$ \\
\hline \multicolumn{3}{|c|}{ Degree of communication with doctors } \\
\hline Very well $(n=132)$ & $81(61.4)$ & $52.50,69.71$ \\
\hline Well $(n=362)$ & $116(32.0)$ & $27.26,37.12$ \\
\hline Neither $(n=189)$ & $16(8.5)$ & $4.92,13.38$ \\
\hline Poor $(n=80)$ & $4(5.0)$ & $1.38,12.31$ \\
\hline Very poor $(n=14)$ & $0(0.0)$ & $0.00,23.16$ \\
\hline \multicolumn{3}{|c|}{ Established treatment goal } \\
\hline Yes $(n=476)$ & $156(32.8)$ & $28.57,37.19$ \\
\hline No $(n=301)$ & $61(20.3)$ & $15.87,25.26$ \\
\hline \multicolumn{3}{|c|}{ Improvement of ADL by treatment for bilateral foot pain } \\
\hline Very well $(n=58)$ & $45(77.6)$ & $64.73,87.49$ \\
\hline Well $(n=157)$ & $91(58.0)$ & $49.83,65.78$ \\
\hline Neither $(n=427)$ & $71(16.6)$ & $13.22,20.50$ \\
\hline Poor $(n=117)$ & $10(8.5)$ & $4.17,15.16$ \\
\hline Very poor $(n=18)$ & $0(0.0)$ & $0.00,18.53$ \\
\hline
\end{tabular}

Improvement of QOL by treatment for bilateral foot pain

$\begin{array}{lcl}\text { Very well }(n=40) & 31(77.5) & 61.55,89.16 \\ \text { Well }(n=163) & 99(60.7) & 52.79,68.28 \\ \text { Neither }(n=438) & 78(17.8) & 14.34,21.72 \\ \text { Poor }(n=107) & 8(7.5) & 3.28,14.20 \\ \text { Very poor }(n=29) & 1(3.4) & 0.09,17.76\end{array}$

Preference for bilateral foot pain treatment

Receive stronger treatment $34(13.7) \quad 9.65,18.56$ $(n=249)$

Continue current treatment 166 (36.5) 32.05, 41.09 $(n=455)$

Receive weaker treatment $17(23.3) \quad 14.19,34.65$ $(n=73)$

$A D L$ actions in daily life, $C I$ confidence interval, $Q O L$ quality of life

a The value reported for "Satisfied" is the sum of "Very satisfied" and "Satisfied" responses satisfied with treatment than those who answered "yes" to fewer than three questions (25.5\% vs $29.8 \%)$. For each symptom of pain (according to the DN4), except for itching, satisfaction was higher among participants who answered "no" compared with those who answered "yes". Those who reported experiencing a feeling of pins and needles were least satisfied with treatment $(21.2 \%)$ compared with participants who reported numbness (27.3\%), itching $(29.2 \%)$, or all other symptoms of pain (approximately 25\% satisfaction for each).

Satisfaction with treatment according to the test used for diagnosis and timing of the test is shown in the Supplementary Material (Supplemental Table 2). There were no differences in satisfaction with treatment between participants who were or were not diagnosed using vibration sensation or autonomic nervous function tests. For all other testing methods, participants who had been diagnosed with each test had a higher level of satisfaction with treatment than those who had not been diagnosed. There were no notable differences in satisfaction with treatment based on the timing of diagnostic tests.

Comprehensive survey results according to participant and disease characteristics are shown in the Supplementary Material (Supplemental Table 3).

\section{DISCUSSION}

This observational, web-based study examined satisfaction of treatment received for bilateral foot pain in participants with diabetes who experienced pain or numbness in both feet. As expected, the participant group in our study was similar to a previously published questionnaire study of patients with DPNP in regard to age (50-79 years: both studies, about $80 \%$ ), gender (male: both studies, about 70\%), and HbA1c value $(<7.0 \%$ : both studies, about $50 \%)$ [29]. Nearly half of the participants had an HbA1c value of $<7.0 \%$, indicating that many participants had good control of their diabetes; however, participant satisfaction was low $(27.9 \%)$, and participants may not have been adequately treated for DPNP. 
Table 4 Satisfaction with treatment by DN4 questionnaire answers

\begin{tabular}{lll}
\hline & \multicolumn{3}{l}{ Satisfied $^{\mathrm{a}}$} & \\
\cline { 2 - 2 }$n(\%)$ & $95 \% \mathrm{CI}$ \\
\hline
\end{tabular}

DN4 score

$$
\begin{array}{lrl}
\geq 3(n=337) & 86(25.5) & 20.95,30.53 \\
<3(n=440) & 131(29.8) & 25.54,34.28
\end{array}
$$

DN4 score of participants who were treated or untreated for pain

$$
\begin{array}{lll}
\geq 3 \text { and treated }(n=225) & 64(28.4) & 22.65,34.82 \\
\geq 3 \text { and untreated }(n=112) & 22(19.6) & 12.74,28.22 \\
<3 \text { and treated }(n=249) & 81(32.5) & 26.75,38.73 \\
<3 \text { and untreated }(n=191) & 50(26.2) & 20.10,33.01
\end{array}
$$

Burning sensation

$$
\begin{array}{lrl}
\text { Yes }(n=99) & 25(25.3) & 17.06,34.98 \\
\text { No }(n=678) & 192(28.3) & 24.95,31.87
\end{array}
$$

Painful cold

$$
\begin{aligned}
& \text { Yes }(n=245) \\
& \text { No }(n=532)
\end{aligned}
$$$$
63(25.7) \quad 20.36,31.67
$$$$
154(28.9) \quad 25.13,33.00
$$

Electric shocks

\begin{tabular}{|c|c|c|}
\hline & \multicolumn{2}{|l|}{ Satisfied $^{\mathbf{a}}$} \\
\hline & $n(\%)$ & $95 \% \mathrm{CI}$ \\
\hline No $(n=616)$ & $170(27.6)$ & $24.10,31.31$ \\
\hline
\end{tabular}

$$
\begin{aligned}
& \text { Yes }(n=319) \\
& \text { No }(n=458)
\end{aligned}
$$

$82(25.7) \quad 21.00,30.87$

$135(29.5) \quad 25.34,33.88$

Tingling

$$
\begin{aligned}
& \text { Yes }(n=380) \\
& \text { No }(n=397)
\end{aligned}
$$$$
97(25.5) \quad 21.22,30.22
$$$$
120(30.2) \quad 25.75,35.01
$$

Pins and needles

$$
\begin{aligned}
& \text { Yes }(n=170) \\
& \text { No }(n=607)
\end{aligned}
$$$$
36(21.2) \quad 15.29,28.09
$$$$
181(29.8) \quad 26.20,33.63
$$

Numbness

$$
\begin{aligned}
& \text { Yes }(n=587) \\
& \text { No }(n=190) \\
& \text { Itching } \\
& \text { Yes }(n=161)
\end{aligned}
$$

$160(27.3) \quad 23.69,31.05$

$57(30.0) \quad 23.58,37.06$

$47(29.2) \quad 22.30,36.87$
Table 4 continued

Participants who were suspected of having DPN based on answering "yes" to at least three questions on the DN4 questionnaire (43.4\%) had a lower level of satisfaction compared with those who answered "yes" to fewer than three questions. Satisfaction with treatment was particularly low (19.6\%) in untreated participants with a DN4 score of at least 3. This may mean that participants with suspected DPNP need more appropriate treatment for their pain.

The factors that had the greatest influence on satisfaction with treatment were improvement in ADL, improvement in QOL, and communication with doctors. A study of satisfaction with treatment in patients who were treated for chronic pain with a nonsteroidal anti-inflammatory or neuropathic pain drug reported similar findings [21]. In that study, satisfaction with treatment was higher in patients who reported improved pain relief, improved ADL, and good communication with their doctor.

A study conducted in Japan found that 93.5\% of participants with diabetes who had sensory symptoms in both legs had an abnormal Achilles tendon reflex [30]. The findings of that study indicate that Achilles tendon reflex evaluation should be conducted when patients are screened for DPN. Although it was the most common test used to diagnose DPN in our study, followed by autonomic nerve function, pain sensation, vibration sensation, and nerve conduction tests, the implementation rates of these tests were low. Less than half of the participants reported receiving each of the diagnostic tests. An online survey conducted in the USA reported that $64 \%$ of healthcare 
professionals never had their patients complete a DPN assessment questionnaire and only $41 \%$ performed specific tests on all patients who reported having symptoms of DPN [31]. Additionally, a cross-sectional study conducted in the USA by Schumacher et al. reported that, of the 71 study participants, only $22 \%$ reported having a diagnosis of DPN even though 54\% reported having symptoms of pain in their hands, arms, legs, or feet [32]. These results suggest that the diagnosis rate of DPN is low and that DPN is not only underdiagnosed in Japan but also in the USA. We found that many participants were tested when they complained of symptoms, including pain and numbness, indicating that communication of symptoms may be an important trigger for testing. The present survey reported no difference in satisfaction with treatment between those who had been diagnosed with DPN and those who had not, suggesting that diagnosis alone did not lead to a higher level of satisfaction with treatment.

While the most frequent type of pain treatment was oral medication, $46.3 \%$ of participants received no treatment $(39.0 \%)$ or nonpharmacotherapy (7.3\%). These participants had a lower satisfaction with treatment compared with those who received other treatments. Regardless, satisfaction with treatment did not exceed $40 \%$ for any of the treatment methods. These results indicate that treatment type and method may influence patient satisfaction and should be considered when making treatment decisions.

A study conducted in the USA reported that the most common symptom of pain among participants with painful DPN $(n=20)$ was numbness; this was also true for the present study, though the rate of this symptom was quite different between studies (Wiklund et al. vs present study, $40 \%$ vs $75.5 \%$ ) [33]. Additionally, $60 \%$ of participants in the study by Wiklund et al. reported that their symptoms affected their ability to do activities, while $72.3 \%$ of participants reported the same issue (i.e., answered "neither", "poor", or "very poor" to satisfaction with treatment Q15: improvement of ADL by treatment for bilateral foot pain) in the present study. Another survey that included participants with diabetes and painful DPN $(n=31)$ reported that $61.3 \%$ noted an impact on their QOL, though $64.5 \%$ of participants reported that this pain did not prevent them from participating in activities [34]. This survey also reported that, of the 15 participants who were currently being treated for nerve pain, $66.7 \%$ were satisfied with their treatment. This contrasts with the $27.9 \%$ reported in the present study; however, it should be noted that not all participants in the present study were receiving treatment for their DPNP. While there are some differences between studies, these are likely due to differences in participant populations. The present study limited participants to those with pain in the lower limbs of both feet who had consulted a physician regarding DPNP, while the other two studies did not. Additionally, the present study included substantially more participants.

As previously noted, communication between participant and doctor had a great impact on whether the participant was satisfied with treatment. In relation to this, having an established treatment goal also positively affected satisfaction with treatment. Together, these findings highlight the importance of good patient-doctor communication. Our findings of low overall satisfaction with treatment and the strong influence of patient-doctor communication on satisfaction with treatment are in line with the findings of the US survey study, suggesting a need for improved communication regarding management of DPNP between patients and their doctors [31]. Poor communication between participants with type 2 diabetes and their doctors was also noted in a Japanese survey study (2017-2018) evaluating factors associated with reluctance to initiate or continue oral antihyperglycemic agent (OAHA) treatment for type 2 diabetes [35]. The researchers reported a low to moderate level of satisfaction with how physicians presented treatment options (newly diagnosed, 17.3\%; current OAHA users, 47.2\%; participants who discontinued OAHA, 47.6\%). Additionally, this survey found that more than $50 \%$ of participants were unaware that neuropathic pain was a potential complication of type 2 diabetes. After learning this information, $34.8 \%$ of 
respondents reported that they were likely to start or restart OAHA treatment. This study further demonstrates the issues around patient-doctor communication and appropriate treatment for diabetes.

Despite the inherent limitations of webbased surveys, they give researchers access to large numbers of participants and the ability to collect data over a short period of time. Additionally, survey data are relatively straightforward to analyze. Overall, surveys, such as the one utilized in the present study, can provide broad and valuable overviews of data. In fact, several web-based surveys have been conducted to obtain data related to the burden of diabetes and DPNP among different populations of participants with diabetes [18, 29, 35-39].

Our study had several limitations. First, answers to the web-survey questionnaire were based on information provided by the participants and were not confirmed by a doctor. Second, a web-based questionnaire may have led to respondent bias. Finally, participant background was limited to the disease panel used to identify potential respondents.

\section{CONCLUSIONS}

Participants with diabetes who experience pain or numbness in both feet reported a low level of satisfaction with treatment for bilateral foot pain and would greatly benefit from improved communication with doctors, receiving a diagnosis of DPN using appropriate clinical tests, and receiving appropriate treatment for their pain.

\section{ACKNOWLEDGEMENTS}

We would like to thank the study participants for their valuable contribution by taking part in this study.

Funding. This study, as well as the Open Access and Rapid Service fees for publication, were funded by Daiichi Sankyo Co., Ltd., Tokyo, Japan.
Medical Writing and Editorial Assistance. The authors thank Hideyuki Sasaki, MD, $\mathrm{PhD}$, of Kansai University of Health Sciences for consultation and advice on research concepts; and Michael LoPresti, MS, of INTAGE Healthcare Inc. for reviewing the manuscript. The authors thank Sarah Bubeck, PhD, of Edanz Pharma for providing medical writing support, which was funded by Daiichi Sankyo Co., Ltd., Tokyo, Japan, in accordance with Good Publication Practice (GPP3) guidelines (http://www. ismpp.org/gpp3).

Authorship. All named authors meet the International Committee of Medical Journal Editors (ICMJE) criteria for authorship for this article, take responsibility for the integrity of the work as a whole, and have given their approval for this version to be published.

Disclosures. Takahisa Deguchi received lecture, consultant, and manuscript writing fees from Daiichi Sankyo Co., Ltd. Hiroshi Takatsuna, Mizuka Yokoyama, and Kazuhito Shiosakai are employees of Daiichi Sankyo Co., Ltd. Teruyoshi Inoue is a former employee of Daiichi Sankyo Co., Ltd. Haruhiko Seki and Yoshiyuki Uetake are employees of INTAGE Healthcare Inc. Daiichi Sankyo Co., Ltd. participated in study design, decision to publish, and preparation of the manuscript. INTAGE Healthcare Inc. participated in data collection and analysis, preparation of the manuscript, study design, and decision to publish. The authors have no other relevant affiliations or financial involvement with any organization or entity with a financial interest in or financial conflict with the subject matter or materials discussed in the manuscript apart from those disclosed. Teruyoshi Inoue was retired at the time of submission.

Compliance with Ethics Guidelines. The study protocol was approved by the ethics review board at the Kitamachi Clinic (approval number: not specified) prior to conducting the study (August 19, 2020). All procedures were conducted in accordance with the ethical standards of the responsible committee on human experimentation, both institutional and 
national, and/or with the Helsinki Declaration of 1964 and later versions. This study was also conducted in accordance with the Japanese ethical guidelines for medical and health research involving human subjects, the Act on the Protection of Personal Information, and the revised Personal Information Protection Act. All participants provided electronic informed consent.

Data Availability. The datasets generated during and/or analyzed during the current study are available from the corresponding author on reasonable request.

Author Contributions. Takahisa Deguchi: conceptualization, methodology, writingoriginal draft, writing-review and editing. Hiroshi Takatsuna: conceptualization, methodology, project administration, writing-original draft, writing-review and editing. Mizuka Yokoyama: conceptualization, methodology, writing-original draft, writing-review and editing. Kazuhito Shiosakai: conceptualization, methodology, writing-original draft, writingreview and editing. Teruyoshi Inoue: conceptualization, methodology, writing-original draft, writing-review and editing. Haruhiko Seki: methodology, project administration, software, writing-review and editing. Yoshiyuki Uetake: formal analysis, software, validation, writing-review and editing.

Open Access. This article is licensed under a Creative Commons Attribution-NonCommercial 4.0 International License, which permits any non-commercial use, sharing, adaptation, distribution and reproduction in any medium or format, as long as you give appropriate credit to the original author(s) and the source, provide a link to the Creative Commons licence, and indicate if changes were made. The images or other third party material in this article are included in the article's Creative Commons licence, unless indicated otherwise in a credit line to the material. If material is not included in the article's Creative Commons licence and your intended use is not permitted by statutory regulation or exceeds the permitted use, you will need to obtain permission directly from the copyright holder. To view a copy of this licence, visit http://creativecommons.org/licenses/bync/4.0/.

\section{REFERENCES}

1. Vinik AI, Nevoret ML, Casellini C, Parson H. Diabetic neuropathy. Endocrinol Metab Clin North Am. 2013;42:747-87.

2. Gylfadottir SS, Weeracharoenkul D, Andersen ST, Niruthisard S, Suwanwalaikorn S, Jensen TS. Painful and non-painful diabetic polyneuropathy: clinical characteristics and diagnostic issues. J Diabetes Investig. 2019;10:1148-57.

3. Gore M, Brandenburg NA, Dukes E, Hoffman DL, Tai KS, Stacey B. Pain severity in diabetic peripheral neuropathy is associated with patient functioning, symptom levels of anxiety and depression, and sleep. J Pain Symptom Manag. 2005;30:374-85.

4. Tesfaye S, Vileikyte L, Rayman G, et al. Painful diabetic peripheral neuropathy: consensus recommendations on diagnosis, assessment and management. Diabetes Metab Res Rev. 2011;27:629-38.

5. Girach A, Julian TH, Varrassi G, Paladini A, Vadalouka A, Zis P. Quality of life in painful peripheral neuropathies: a systematic review. Pain Res Manag. 2019;2019:2091960.

6. Pop-Busui R, Boulton AJ, Feldman EL, et al. Diabetic neuropathy: a position statement by the American Diabetes Association. Diabetes Care. 2017;40: 136-54.

7. Gregg EW, Gu Q, Williams D, et al. Prevalence of lower extremity diseases associated with normal glucose levels, impaired fasting glucose, and diabetes among U.S. adults aged 40 or older. Diabetes Res Clin Pract. 2007;77:485-8.

8. Alleman CJ, Westerhout KY, Hensen $\mathrm{M}$, et al. Humanistic and economic burden of painful diabetic peripheral neuropathy in Europe: a review of the literature. Diabetes Res Clin Pract. 2015;109: $215-25$

9. Satoh J, Baba M, Yagihashi S, et al. Frequency of diabetic polyneuropathy (DPN) and clinical significance of Achilles tendon reflex in diagnosis of DPN. J Japan Diabetes Soc. 2007;50:799-806 (in Japanese).

10. Goto Y. Research about diabetic neuropathy. J Jpn Physicians Assoc. 2002;17:125-38 (in Japanese). 
11. Yokoyama H, Tsuji T, Hayashi S, Kabata D, Shintani A. Factors associated with diabetic polyneuropathyrelated sensory symptoms and signs in patients with polyneuropathy: a cross-sectional Japanese study (JDDM 52) using a non-linear model. J Diabetes Investig. 2020;11:450-7.

12. American Diabetes Association. 11. Microvascular complications and foot care: standards of medical care in diabetes-2020. Diabetes Care. 2020;43: S135-51.

13. Tesfaye S, Boulton AJ, Dyck PJ, et al. Diabetic neuropathies: update on definitions, diagnostic criteria, estimation of severity, and treatments. Diabetes Care. 2010;33:2285-93.

14. The Japanese study group of diabetic neuropathy. Simple diagnostic criteria for diabetic polyneuropathy. Peripheral Nerve. 2006;17:101-3 (in Japanese).

15. Araki E, Goto A, Kondo T, et al. Japanese clinical practice guideline for diabetes 2019. Diabetol Int. 2020;11:165-223.

16. Japan Promotion Council for Diabetes Prevention and Countermeasures. Report on abnormalities in foot appearance and diabetic neuropathy in diabetes in Japan 2008 (in Japanese). https://www. med.or.jp/dl-med/tounyoubyou/diabetes080312. pdf. Accessed Feb 15, 2021.

17. Tsuji M, Yasuda T, Kaneto H, et al. Painful diabetic neuropathy in Japanese diabetic patients is common but underrecognized. Pain Res Treat. 2013;2013:318352.

18. Sasaki H, Takatsuna $H$, Inoue $T$, et al. A cross-sectional survey of patients with suspected diabetic peripheral neuropathic pain in Japan. Intern Med. 2021;60:357-65.

19. Yang M, Qian C, Liu Y. Suboptimal treatment of diabetic peripheral neuropathic pain in the United States. Pain Med. 2015;16:2075-83.

20. Nemoto O, Nakagawa H, Igarashi A, et al. The results of a satisfaction survey concerning treatment for psoriasis patients. Jpn J Dermatol. 2003;14:2059-69.

21. Ushida $\mathrm{T}$, Inoue $\mathrm{T}$, Matsui D, et al. Cross-sectional study of patient satisfaction with oral analgesics in patients with chronic pain in Japan. Expert Opin Pharmacother. 2020;21:983-91.

22. Bouhassira D, Attal N, Alchaar H, et al. Comparison of pain syndromes associated with nervous or somatic lesions and development of a new neuropathic pain diagnostic questionnaire (DN4). Pain. 2005;114:29-36.
23. Matsuki Y, Sukenaga N, Miyagi K, et al. Reliability and validity of the Japanese translation of the DN4 Diagnostic Questionnaire in patients with neuropathic pain. J Anesth. 2018;32:403-8.

24. Japan Ministry of Heath Labour and Welfare. Ethical guidelines for medical and health research involving human subjects 2015. https://www. mhlw.go.jp/file/06-Seisakujouhou-10600000Daijinkanboukouseikagakuka/0000080278.pdf. Accessed Feb 15, 2021.

25. INTAGE Inc. Home page. https://www.intage.co.jp/ english/. Accessed Feb 15, 2021.

26. Rakuten Insight, Inc. https://insight.rakuten.co.jp/ en/. Accessed Feb 15, 2021.

27. Japan Ministry of Health Labour and Welfare. Patient Survey Volume 1 (Nationwide); Table 62total number of patients, gender/age group $\times$ injury and illness subcategory 2017 (in Japanese). https:// www.e-stat.go.jp/dbview?sid=0003318621. Accessed March 4, 2021.

28. Japan Diabetes Society. Treatment guide for diabetes 2016-2017. Tokyo: Bunkodo; 2016. http:// www.fa.kyorin.co.jp/jds/uploads/Treatment_ Guide_for_Diabetes_2016-2017.pdf. Accessed Feb $15,2021$.

29. Ono $\mathrm{H}$, Inoue $\mathrm{T}$, Hatakeyama $\mathrm{N}$, et al. Patient questionnaire survey on diabetic neuropathic pain. Prog Med. 2008;28:1199-206 (in Japanese).

30. Sugimoto K, Suzuki S. Validation of a simple screening questionnaire on sensory symptoms for diabetic neuropathy. J Jpn Diabetes Soc. 2016;59: 360-8 (in Japanese).

31. Sadosky A, Hopper J, Parsons B. Painful diabetic peripheral neuropathy: results of a survey characterizing the perspectives and misperceptions of patients and healthcare practitioners. Patient. 2014;7:107-14.

32. Schumacher C, Glosner SE. Assessment of pain and impact of care among patients with painful diabetic peripheral neuropathy. J Am Pharm Assoc. 2003;2014(54):14-8.

33. Wiklund I, Holmstrom S, Stoker M, Wyrwich KW, Devine M. Are treatment benefits in neuropathic pain reflected in the Self Assessment of Treatment questionnaire? Health Qual Life Outcomes. 2013;11:8.

34. Dulipsingh L, Zailskas S, Goldsby T, McInnis T, Marotta A. Assessment of pain and treatment satisfaction in patients with painful diabetic peripheral neuropathy. Conn Med. 2013;77:523-7. 
35. Kubo T, Okuyama K, Zhao X, Singh SS, Tokita S. Factors associated with reluctance to initiate or continue oral antihyperglycemic agent (OAHA) treatments in type 2 diabetes mellitus patients in Japan: an observational patient-reported study. Diabetes Metab Syndr. 2019;13:1201-7.

36. Reach G, Le Pautremat V, Gupta S. Determinants and consequences of insulin initiation for type 2 diabetes in France: analysis of the National Health and Wellness Survey. Patient Prefer Adherence. 2013;7:1007-23.
37. Cepeda MS, Wilcox M, Levitan B. Pain qualities and satisfaction with therapy: a survey of subjects with neuropathic pain. Pain Med. 2013;14:1745-56.

38. Lopez JM, Bailey RA, Rupnow MF, Annunziata K. Characterization of type 2 diabetes mellitus burden by age and ethnic groups based on a nationwide survey. Clin Ther. 2014;36:494-506.

39. Van Acker K, Bouhassira D, De Bacquer D, et al. Prevalence and impact on quality of life of peripheral neuropathy with or without neuropathic pain in type 1 and type 2 diabetic patients attending hospital outpatients clinics. Diabetes Metab. 2009;35:206-13. 